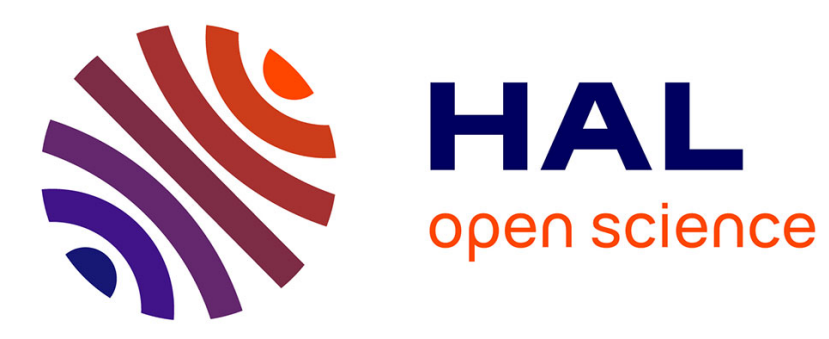

\title{
Universal dimensional crossover of domain wall dynamics in ferromagnetic films
}

W. Savero Torres, R Díaz Pardo, S. Bustingorry, A. Kolton, Aristide

Lemaitre, V. Jeudy

\section{- To cite this version:}

W. Savero Torres, R Díaz Pardo, S. Bustingorry, A. Kolton, Aristide Lemaitre, et al.. Universal dimensional crossover of domain wall dynamics in ferromagnetic films. Physical Review B: Condensed Matter and Materials Physics (1998-2015), 2019, 99 (20), 10.1103/PhysRevB.99.201201 . hal-02352304

\section{HAL Id: hal-02352304 \\ https://hal.science/hal-02352304}

Submitted on 6 Nov 2019

HAL is a multi-disciplinary open access archive for the deposit and dissemination of scientific research documents, whether they are published or not. The documents may come from teaching and research institutions in France or abroad, or from public or private research centers.
L'archive ouverte pluridisciplinaire HAL, est destinée au dépôt et à la diffusion de documents scientifiques de niveau recherche, publiés ou non, émanant des établissements d'enseignement et de recherche français ou étrangers, des laboratoires publics ou privés. 


\title{
Universal dimensional crossover of domain wall dynamics in ferromagnetic films
}

\author{
W. Savero Torres, ${ }^{1}$ R. Díaz Pardo, ${ }^{1}$ S. Bustingorry, ${ }^{2}$ A. B. Kolton, ${ }^{3}$ A. Lemaître,,${ }^{4}$ and V. Jeudy ${ }^{1, *}$ \\ ${ }^{1}$ Laboratoire de Physique des Solides, Université Paris-Sud, Université Paris-Saclay, CNRS, UMR8502, 91405 Orsay, France \\ ${ }^{2}$ Instituto de Nanociencia y Nanotecnología, CNEA-CONICET, Centro Atómico Bariloche, R8402AGP San Carlos de Bariloche, \\ Río Negro, Argentina \\ ${ }^{3}$ CONICET and Instituto Balseiro (UNCu), Centro Atómico Bariloche, 8400 San Carlos de Bariloche, Río Negro, Argentina \\ ${ }^{4}$ Centre de Nanosciences et de Nanotechnologies, CNRS, Univ. Paris-Sud, Université Paris-Saclay, 91120 Palaiseau, France
}

(Received 1 December 2018; revised manuscript received 13 March 2019; published 6 May 2019)

\begin{abstract}
The magnetic domain wall motion driven by a magnetic field is studied in $(\mathrm{Ga}, \mathrm{Mn}) \mathrm{As}$ and $(\mathrm{Ga}, \mathrm{Mn})(\mathrm{As}, \mathrm{P})$ films of different thicknesses. In the thermally activated creep regime, a kink in the velocity curves and a jump of the roughness exponent evidence a dimensional crossover in the domain wall dynamics. The measured values of the roughness exponent $\zeta_{1 d}=0.62 \pm 0.02$ and $\zeta_{2 d}=0.45 \pm 0.04$ are compatible with theoretical predictions for the motion of elastic line $(d=1)$ and surface $(d=2)$ in two- and three-dimensional media, respectively.
\end{abstract}

DOI: 10.1103/PhysRevB.99.201201

\section{INTRODUCTION}

\section{A. Universal dynamical behaviors}

Driven elastic interfaces in disordered media present intriguing scaling behaviors determined by properties such as the symmetry [1], the range of elastic interactions [2,3], the correlations of disorder [4], and the dimensionality [4,5]. Experimental situations are often complex and a given dynamical system can present crossovers between different scaling behaviors, corresponding to different universality classes. Some examples of this complexity are the coexistence of two distinct critical dynamics at different length scales [6] reported in crack propagation [7], the crossover due to variable interaction range in ferromagnets [2], or to finite size effects in ferromagnetic nanowires $[5,8]$ to name a few. Investigation of universal crossovers induced by different characteristic length scales and particularly by finite size effects are a key for understanding interface dynamics. It is also of significant technological interest for nanodevices based on domain wall (DW) motion $[9,10]$.

\section{B. Roughness and creep exponents}

Evidencing a universal dimensional crossover experimentally is rather challenging and ultrathin and thin ferromagnetic films with perpendicular anisotropy [11] offer the opportunity to perform combined studies of interface dynamics and roughness. In this system, the DW creep motion is controlled by pinning energy barriers, thermal activation, interface elasticity, and the driving force, $f$, which can be due to magnetic field $(f \propto H)[4,11,12]$. Below the depinning threshold $\left(H<H_{d}\right)$, the velocity follows an Arrhenius law $\ln v \sim-\Delta E / k_{B} T$ with an effective energy barrier decreasing with the drive $\Delta E \sim$ $H^{-\mu} . k_{B} T$ and $\mu$ are the thermal activation energy and the so-called universal creep exponent, respectively. The thermal

*vincent.jeudy@u-psud.fr activation produces compact jumps (so-called thermal nuclei) allowing the interface to overcome pinning barriers and to advance in the direction of the drive. The pattern of motion consists in successive avalanches composed of many thermally activated nuclei with a broad size distribution [13]. The largest nuclei sizes, of order $L_{\mathrm{opt}}$, are predicted to decrease with increasing the drive $[4,11,12]$ as $L_{\text {opt }} \sim H^{-1 /\left(2-\zeta_{\text {eq }}\right)}$, with $\zeta_{\text {eq }}$ the universal roughness exponent of the DW at the equilibrium $(H=0)$. The roughness and creep exponents are linked by the scaling relation $\mu=\left(d-2+2 \zeta_{\mathrm{eq}}\right) /\left(2-\zeta_{\mathrm{eq}}\right)$, with $d$ the dimension of the interface, whose experimental verification is a stringent test of theory $[4,11]$.

\section{Finite size effects}

Since the thermal nuclei with the largest sizes/barriers ultimately controls the interface velocity in the creep regime [13], finite size effects are expected to occur at sufficient low drive when $L_{\text {opt }}(H)$ becomes larger than one length scale of the embedding medium. For ultrathin films [11], the optimum length $L_{\text {opt }}(H)$ always remains larger than the film thickness $t(<1 \mathrm{~nm})$. The creep and roughness exponents deduced from experiments are compatible with mean-field theoretical predictions $\left(\mu=1 / 4\right.$ and $\left.\zeta_{\text {eq }}=2 / 3\right)$ for an elastic line $(d=1)$ moving in a two-dimensional medium $(D=2)$. For ultrathin nanowires, the increase of $L_{\mathrm{opt}}(H)$ above the nanowire width with decreasing drive was shown to result in a dimensional crossover between the elastic line behavior $(d=1, D=2)$ and a motion similar to particle hopping $(d=0)$ along a line $(D=1)$ [5]. Surprisingly, the two-dimensional behavior of DWs $(d=2, D=3)$, detected by Barkhaussen well before the development of nanotechnologies, is less well understood. Flow dynamics [14] and universal behaviors of the depinning transition $[15,16]$ have been investigated. However, to the best of our knowledge, the two-dimensional behavior of DW creep motion has not been evidenced yet. 


\section{Dimensional crossover}

In this Rapid Communication, we report on evidences of two-dimensional behavior of DWs creep motion and dimensional crossover due to the ferromagnetic film finite thickness. We first show that below and above a crossover field the velocity curves follow the creep law $\ln v \sim H^{-\mu}$. Below the crossover field, $\mu=1 / 4$ as expected for the motion of an elastic line $(d=1, D=2)$ and above, $\mu=1 / 2$ as predicted for the motion of a surface $(d=2, D=3)$ [11]. A more stringent signature of the dimensional crossover is a jump of the roughness exponent $\zeta$ between two values, which are found in agreement with theoretical predictions for the quenched Edwards-Wilkinson (qEW) model with random-bond shortrange pinning and including anharmonic correction to DW elastic energy [17].

\section{MEASUREMENTS}

\section{A. Experimental methods}

The experiments were performed on three films: $\left(\mathrm{Ga}_{0.95} \mathrm{Mn}_{0.05}\right)\left(\mathrm{As}_{0.9}, \mathrm{P}_{0.1}\right), \quad\left(\mathrm{Ga}_{0.92} \mathrm{Mn}_{0.08}\right)\left(\mathrm{As}_{0.89}, \mathrm{P}_{0.11}\right)$, and $\left(\mathrm{Ga}_{0.93} \mathrm{Mn}_{0.07}\right)$ As of thickness $(t=)$ 12.5, 50, and $80 \mathrm{~nm}$, respectively. The films are single crystals grown by molecular beam epitaxy on a (001) GaAs substrate. The $(\mathrm{Ga}, \mathrm{Mn})(\mathrm{As}, \mathrm{P})$ films were directly deposited onto the substrate, while the $(\mathrm{Ga}, \mathrm{Mn})$ As film was grown onto a relaxed $(\mathrm{Ga}, \mathrm{In}) \mathrm{As}$ interlayer [18]. All the films present an out-of-plane easy magnetization axis. After annealing, the film Curie temperatures were 74,130 , and $126 \mathrm{~K}$, respectively. The pinning is usually due to defects in the materials, which produce fluctuations of domain wall energy. However, the specific nature of those defects in (Ga,Mn)As and $(\mathrm{Ga}, \mathrm{Mn})(\mathrm{As}, \mathrm{P})$ remains an open question (spatial fluctuations of $\mathrm{Mn}$ concentration, misfit dislocations for (Ga,Mn)As [18], fluctuation of the film thickness...).

An optical helium flow cryostat was used to vary the temperature down to $4.3 \mathrm{~K}$. DW motion was observed with a magneto-optical Kerr microscope. The DW displacement was induced by magnetic field pulses (duration: $1 \mu \mathrm{s}$ to $1 \mathrm{~s}$ ) generated by a small coil (diameter $\approx 1 \mathrm{~mm}$, rise time $\approx$ $200 \mathrm{~ns}$ ) directly placed on the films. For each pulse amplitude, the duration was adjusted to obtain a sufficiently large displacement for an accurate velocity measurement without expelling the DW from the field of view. The velocity is defined as the ratio between displacement and pulse duration [11,19-21].

\section{B. Domain wall dynamics}

The different regimes of DW motion driven by magnetic field are presented in Fig. 1 for two different film thicknesses. The depinning threshold $H_{d}(T)$ (indicated by filled stars) corresponds to the inflection points of the velocity curves [see inset of Fig. 1(a)]. It is the thermally activated creep regime upper boundary (for details on the method, see Ref. [22]). For $H>H_{d}$, the linear, temperature-independent evolution of the velocity with $H$ [shown in the inset of Fig. 1(a)] corresponds to the dissipative flow motion. The measured mobility $[\mathrm{m}=$ $v / H=0.56 \pm 0.02 \mathrm{~m} /(\mathrm{s} \mathrm{mT})]$ is close to the reported value [19] for the asymptotic so-called precessional flow regime.
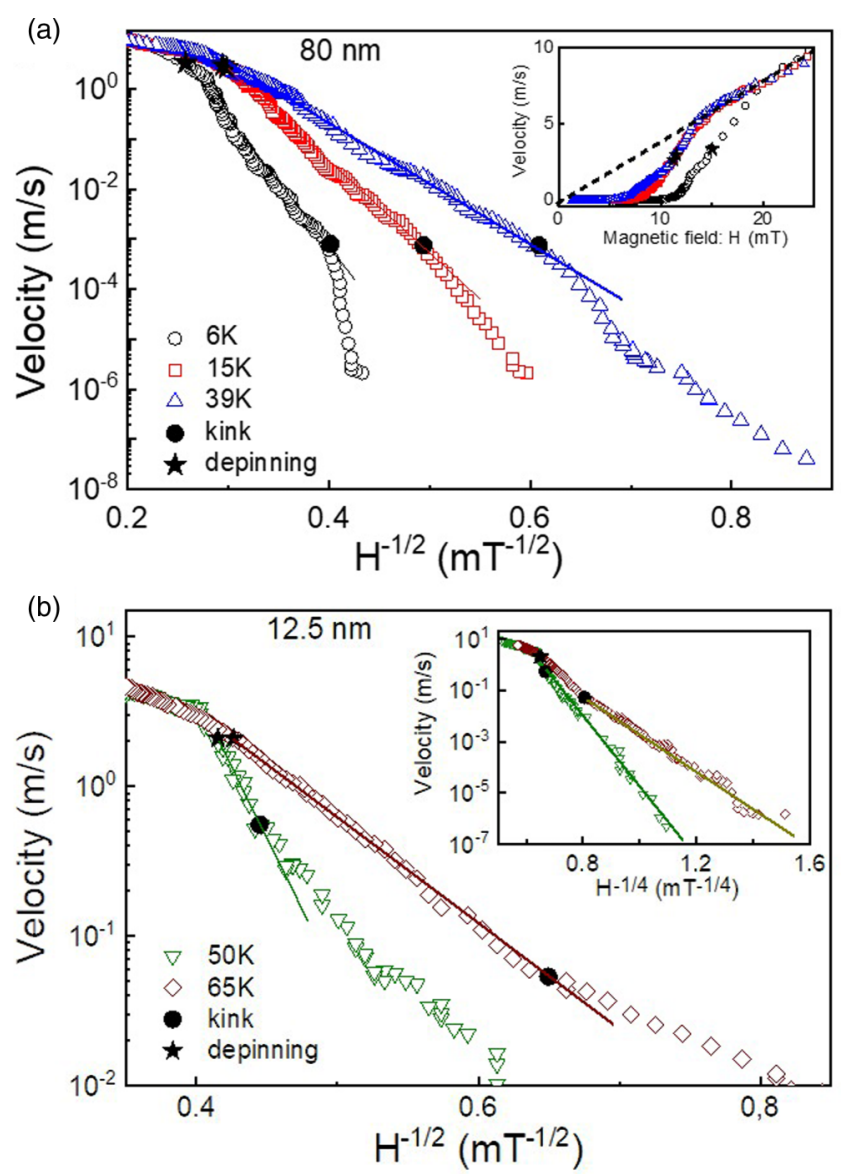

FIG. 1. DW dynamics driven by magnetic field $H$ for (a) an 80-nm-thick (Ga,Mn)As and (b) a 12.5-nm-thick (Ga,Mn)(As,P) film at different temperatures. The semilogarithmic plots of the velocity versus $H^{-\mu}$ with $\mu=1 / 2$ [(a) and (b)] and $\mu=1 / 4$ [inset of (b)] evidence a dimensional crossover in the thermally activated creep regimes. The kinks indicated by filled circles correspond to the crossover field $H_{c}$. The upper limit of the creep regime $H_{d}$ is indicated by stars. The lines are guides for the eyes. The linear plot of the velocity curves [inset of (a)] highlights the linear flow regime (see the dashed line) observed above $H_{d}$. A sliding average over five points was performed to reduce the velocity fluctuations.

In the creep regime $\left[0<H<H_{d}(T)\right]$, the velocity varies with temperature and magnetic field, as expected for a thermally activated motion. Surprisingly, the curves systematically display a kink (indicated by filled circles in Fig. 1) at a well-defined crossover magnetic field $H_{c}(T)$ (see Table I for the values of $H_{c}$ and $H_{d}$ ). The velocity curves follow the creep law $\left(\ln v \sim H^{-\mu}\right.$ ) with $\mu=1 / 4$ for $H<H_{c}(T)$ [see the inset of Fig. 1(b)], and $\mu=1 / 2$ for $H>H_{c}(T)$ [see Figs. 1(a) and 1(b)]. As the values $\mu=1 / 4$ and $\mu=1 / 2$ are expected [11] for the creep motion of an elastic line and a surface, respectively, the critical exponent jump strongly suggests a dimensional crossover of the DW dynamics.

\section{Domain wall roughness}

In order to obtain an independent and more accurate signature of the dimensional crossover, the roughness scaling properties of DWs [11] were analyzed in detail. We calculated 
TABLE I. Crossover $H_{c}$ and depinning $H_{d}$ fields for the three film thicknesses $(t)$ and different temperatures $(T)$. The column "Dynamics" (respectively, "Roughness") corresponds to the kink of velocity curves (see Fig. 1) (respectively, the step in roughness exponent curves; see Fig. 3). The numbers in parentheses correspond to the crossover width.

\begin{tabular}{lcccc}
\hline \hline$t(\mathrm{~nm})$ & $T(\mathrm{~K})$ & $\begin{array}{c}\text { Depinning } \\
H_{d}(\mathrm{mT})\end{array}$ & $\begin{array}{c}\text { Dynamics } \\
H_{c}(\mathrm{mT})\end{array}$ & $\begin{array}{c}\text { Roughness } \\
H_{c}(\mathrm{mT})\end{array}$ \\
\hline 12 & 4.3 & 8.19 & $8.0(0.4)$ & \\
& 10 & 7.97 & $7.6(0.4)$ & \\
& 30 & 7.22 & $6.8(0.4)$ & \\
& 50 & 5.8 & $5.2(0.2)$ & $5.7(0.4)$ \\
& 65 & 5.5 & $3.9(1.6)$ & \\
50 & 30 & 3.30 & $2.4(0.3)$ & \\
& 60 & 3.24 & $1.15(0.20)$ & $1.45(0.15)$ \\
& 90 & 3.22 & $0.67(0.05)$ & $0.82(0.14)$ \\
& 96 & 3.1 & $0.8(0.2)$ & \\
& 6 & 14.7 & $6.3(0.3)$ & \\
& 15 & 11.5 & $4.9(0.4)$ & $5.0(0.4)$ \\
& 39.6 & 11.15 & $2.6(0.6)$ & $2.5(0.3)$ \\
\hline \hline
\end{tabular}

the correlation function of the DW displacement $u$ defined by

$$
w(L)=\left\langle|u(x+L)-u(x)|^{2}\right\rangle,
$$

where $x$ corresponds to a position on the DW and $L$ a distance from $x$ along the DW. In Eq. (1), the symbol $\langle\cdot\rangle$ corresponds to an average of measurements over all the positions $x$. As the displacement scales as $u \sim L^{\zeta}$, the displacement correlation function should follow a power law $w(L) \sim L^{2 \zeta}$, with different values of $\zeta$ for the elastic line $(d=1)$ and surface $(d=2)$ behaviors of DW. Experimentally, the displacements deduced from differential Kerr imaging (see the inset of Fig. 2) were used to determine the displacement correlation function [11] as a function of $L$. Typical results are reported in Fig. 2 in $\log -\log$ scale and effectively reveal a change in the power law for $w(L)$. The distance range $(1-5 \mu \mathrm{m})$ over which the power law changes is narrow compared to usual measurements $[11,23]$ and most probably originates from the DW displacement anisotropy (see the inset of Fig. 2) produced by a small in-plane magnetic anisotropy [24]. Moreover, the curves are grouped in two sets with different slopes, at low $L$ : the slope $(=2 \zeta)$ is higher at low field $(H<5.1 \mathrm{mT})$ than at high field $(H>6.55 \mathrm{mT})$. In order to analyze this observation in more detail, $\zeta$ was measured systematically. Typical variations of $\zeta$ with reduced applied magnetic field $H / H_{d}$ are reported in Fig. 3. As can be seen, the curves present a sigmoidlike shape with upper and lower levels close to the expected values $\zeta \approx 2 / 3(d=1)$ and $\zeta \approx 0.45(d=2)$ [25]. Moreover, the crossover between the two levels occurs over a rather narrow range of magnetic fields $\left(\Delta H_{c} / H_{d} \approx 0.1-0.2\right)$. A crossover field $H_{c}$ can therefore be defined. The obtained values are reported in Table I (with the label "Roughness") for different temperature. As can be seen, a good agreement is obtained with the values of $H_{c}$ (labeled "Dynamics") deduced from the kink of the velocity curves. Therefore, the step of $\zeta$ curves (see Fig. 3) and the kink in the velocity curves (see Fig. 1) depict two signatures of the same DW dimensional crossover between a line $\left(H<H_{c}\right)$ and an elastic surface $\left(H>H_{c}\right)$.

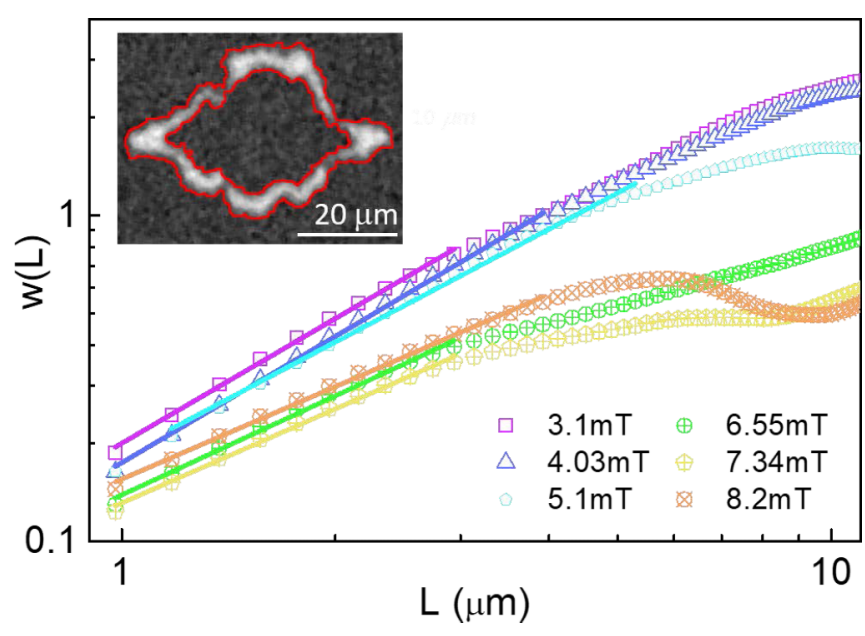

FIG. 2. Displacement correlation function $w$ versus distance $L$ along the DW in log-log scale obtained for different applied magnetic field values for the 80-nm-thick film, at $T=15 \mathrm{~K}$. The lines are fits, for the small distance part, of the scaling relation $w \sim L^{2 \zeta}$ (see text). Two well-separated sets of curves are presenting a different slope. The slope change is observed for $H=5.1-6.55 \mathrm{mT}$ and corresponds to the step of the roughness exponent $\zeta$. Inset: Typical image of a DW displacement (in light gray) deduced from differential Kerr imaging showing two successive DW positions (indicated by the red contours). Pulse duration $50 \mathrm{~ms}$ and amplitude $H=3.6 \mathrm{mT}$, 80-nm-thick film, $T=15 \mathrm{~K}$.

\section{DISCUSSIONS}

\section{A. Thermal nuclei size}

A summary of all the reduced crossover fields $H_{c} / H_{d}$ measured for different film thicknesses is reported in Fig. 4. Interestingly, our measurements provide direct insights on the microscopic length scale involved in the avalanche processes producing DW motion [13]. Indeed, at the crossover field, the maximum excitation length should be close to the film

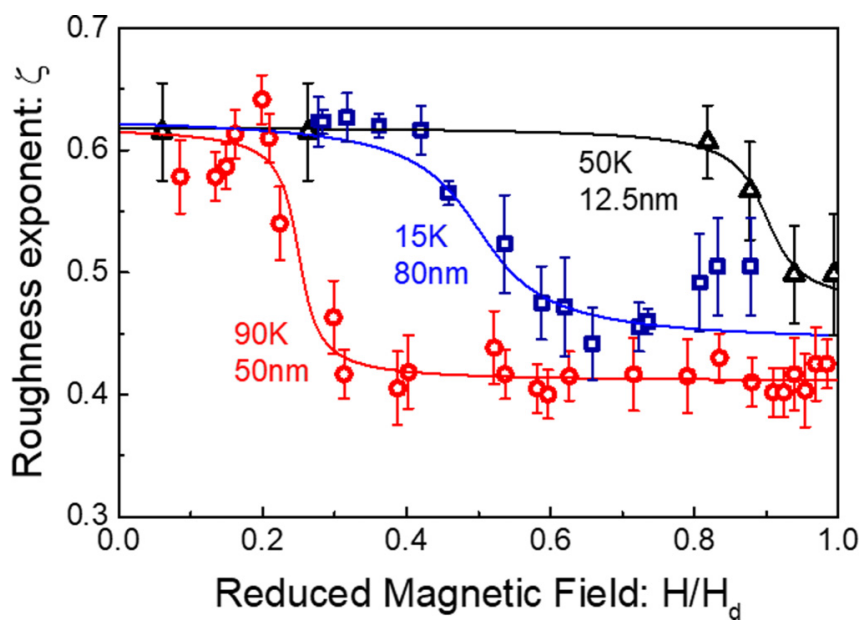

FIG. 3. Roughness exponent $\zeta$ as a function of reduced magnetic field $H / H_{d}$ for three film thicknesses and different temperatures. The steps between two constant $\zeta$ values reflect a dimensional crossover of DW dynamics. The sigmoid lines are guides for the eyes. Each error bar corresponds to the standard deviation of average value. 


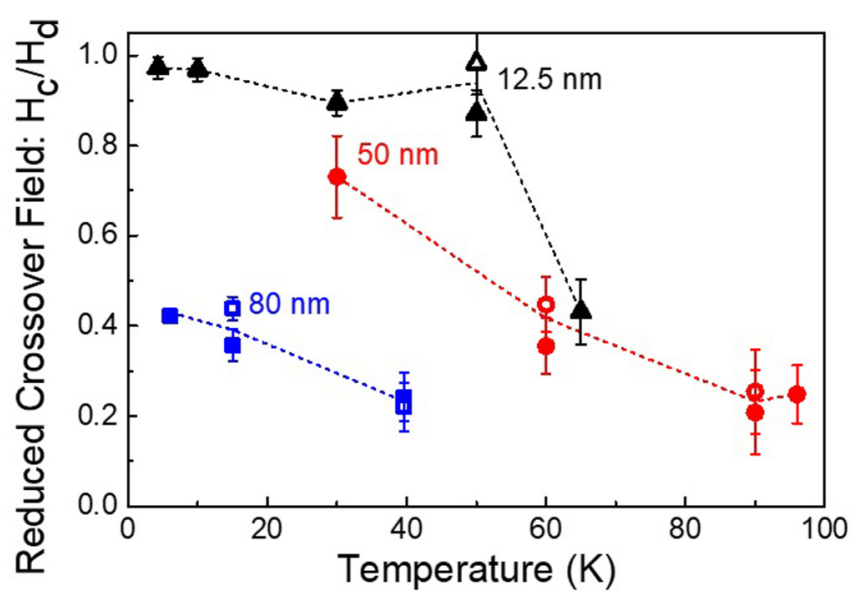

FIG. 4. Temperature variation of the reduced crossover field $H_{c} / H_{d}$ for the three sample thicknesses. The filled and empty symbols correspond to the kink of velocity curves (cf. Fig. 1) and the step of $\zeta$ curves (cf. Fig. 3), respectively. The dimensional crossover is shifted toward the depinning transition $\left(H_{c} / H_{d}=1\right)$ for decreasing film thickness.

thickness $\left[L_{\mathrm{opt}}\left(H_{c}, T\right) \approx t\right]$. Therefore the film thickness $(t=$ $12.5-80 \mathrm{~nm}$ ) gives an order of magnitude of the events triggering magnetization reversal avalanches. A signature of the reduction of $L_{\mathrm{opt}}$ with increasing drive $\left(L_{\mathrm{opt}} \sim H^{-1 /\left(2-\zeta_{\mathrm{eq}}\right)}\right)$ and temperature can be found in Fig. 4. For example, at $T=30 \mathrm{~K}$, $H_{c} / H_{d} \approx 0.3$ (respectively, 0.9) for the 80 (respectively, 12.5) $\mathrm{nm}$, thick films. This indicates that for a fixed temperature, the range $\left(H_{c} / H_{d}<H / H_{d}<1\right)$ over which DW presents a two-dimensional $(d=2, D=3)$ creep motion decreases with decreasing thickness, as expected. Moreover, for a fixed ratio $H_{c} / H_{d}$, the excitation size $L_{\mathrm{opt}}(H, T)$ decreases with increasing temperature, which strongly suggests a decrease of avalanche sizes with increasing temperature.

\section{B. Analysis of the critical exponents}

Let us now discuss the theoretical predictions and experimental results on the roughness exponent in more details. By taking into account all the measurements performed sufficiently away from the crossover, we obtain the average values $\zeta_{1 d}=0.62 \pm 0.02$ and $\zeta_{2 d}=0.45 \pm 0.04$ for $1 d$ and $2 d$ interfaces. The value of $\zeta_{1 d}$ is in good agreement with previous results $[11,20]$ obtained for $\mathrm{Pt} / \mathrm{Co} / \mathrm{Pt}$ ultrathin films for which the DW displacement is isotropic [20,21]. This agreement suggests that the contribution of DW displacements anisotropy (observed the inset of Fig. 2) has a negligible contribution to the roughness.

In ultrathin films, the DW displacement $u(\mathbf{x}, t)$ at position $\mathbf{x}$ and time $t$ driven by an external force $f(\propto H)$ is well described [26] by the following equation:

$$
\frac{\partial u(\mathbf{x}, t)}{\partial t}=-\frac{\partial E_{\mathrm{el}}}{\partial u(\mathbf{x})}+f+v(\mathbf{x}, t)+\eta(\mathbf{x}, u)
$$

where $v(\mathbf{x}, t)$ accounts for thermal noise and $\eta(\mathbf{x}, u)$ for short-range pinning disorder, producing DW roughness. $E_{\mathrm{el}}$ is the elastic energy which tends to flatten the DW. In the case where only the harmonic contribution of $E_{\mathrm{el}}$ is considered, Eq. (2) is reduced to the so-called quenched
Edwards-Wilkinson equation. Theoretically, different roughness exponents are predicted, $\zeta_{\text {eq }}(f \approx 0)$ close to zero drive, and $\zeta_{\text {dep }}\left(f \approx f_{\text {dep }}\right)$ close to the depinning threshold. Moreover, numerical simulations indicate that $L_{\mathrm{opt}}$ is a crossover length scale below (above) which the relevant roughness exponent is $\zeta_{\text {eq }}\left(\zeta_{\text {dep }}\right)$, respectively $[12,13]$. Here, the spatial resolution of magneto-optical Kerr microscopy $(\approx 1 \mu \mathrm{m})$ is well above $L_{\text {opt }}(=12.5-80 \mathrm{~nm})$ for $H=H_{c}$. Increasing $L_{\text {opt }}$ up to $1 \mu \mathrm{m}$ would require one to drive DW at reduced magnetic field values $\left(H / H_{d}\right)$ lower than $2 \times 10^{-2}$, which is one order of magnitude lower than our experimental capabilities (see Fig. 4).

Therefore, the measured roughness exponents have been compared to theoretical predictions for the depinning regime. For an elastic line $(d=1)$ moving in a two-dimensional medium $(D=2)$, analytical calculations [4] and numerical simulation $[17,25]$ predict $\zeta_{\text {dep }}=1.25$ for harmonic $\left(\sim u^{2}\right)$ and $\zeta_{\text {dep }}=0.635 \pm 0.005$ for anharmonic variations of $E_{\mathrm{el}}$. Only the latter prediction is compatible with our experiments $\left(\zeta_{1 d}=\right.$ $0.62 \pm 0.02)$. Note also that the predicted depinning and equilibrium $\left(\zeta_{\text {eq }}=2 / 3\right)$ values are too close to be discriminated experimentally. For the motion of elastic surface $(d=2)$ in a three-dimensional medium $(D=3)$, the predictions are $\zeta_{\text {dep }}=0.75$ for harmonic and $\zeta_{\text {dep }}=0.45$ for anharmonic variations of $E_{\mathrm{el}}$, respectively $[17,25]$. Here also, our experimental results $\left(\zeta_{2 d}=0.45 \pm 0.04\right)$ are only compatible with predictions assuming anharmonic elasticity for the depinning and equilibrium values $\left(\zeta_{\text {eq }}=0.41\right)[25]$.

\section{CONCLUSION}

In conclusion, we have evidenced two concomitant signatures of a dimensional crossover in the thermally activated creep motion of magnetic DWs. On the theoretical front, it would be very interesting to investigate the dimensional crossover and in particular the contribution of anisotropy between the vertical and in-plane direction due to perpendicular anisotropy [14], which should lead to the formation of strongly anisotropic thermal nuclei. Moreover, the DW universal behavior as an elastic surface should be encountered close to the depinning threshold in any films or multilayers with thicknesses larger than a few tens of nanometers [22]. As the motion can be described by a single minimal model ignoring the magnetic structure of DWs, the dimensional crossover should present a universal character and be encountered in other systems than magnets.

\section{ACKNOWLEDGMENTS}

We wish to thank L. Thevenard and C. Gourdon for the loan of the 80-nm-thick sample. S.B. and V.J. acknowledge support by the French-Argentina project ECOS-Sud No. A12E03. This work was also partly supported by the French projects DIM CNano IdF (Region Ile-de-France), the Labex NanoSaclay, No. ANR-10-LABX-0035, and the French RENATECH network. R.D.P. thanks the Mexican council CONACYT for support from PhD fellowship No. 449563. S.B. and A.B.K. acknowledge partial support from Project PIP11220120100250CO (CONICET). 
[1] S. Atis, A. K. Dubey, D. Salin, L. Talon, P. Le Doussal, and K. J. Wiese, Phys. Rev. Lett. 114, 234502 (2015).

[2] K.-S. Ryu, H. Akinaga, and S.-C. Shin, Nat. Phys. 3, 547 (2007).

[3] S. Zapperi, P. Cizeau, G. Durin, and H. E. Stanley, Phys. Rev. B 58, 6353 (1998).

[4] P. Chauve, T. Giamarchi, and P. Le Doussal, Phys. Rev. B 62, 6241 (2000).

[5] K.-J. Kim, J.-C. Lee, S.-M. Ahn, K.-S. Lee, C.-W. Lee, Y. J. Cho, S. Seo, K.-H. Shin, S.-B. Choe, and H.-W. Lee, Nature (London) 458, 740 (2009).

[6] A.-L. Barabási and H. E. Stanley, Fractal Concepts in Surface Growth (Cambridge University Press, Cambridge, UK, 1995).

[7] S. Santucci, M. Grob, R. Toussaint, J. Schmittbuhl, A. Hansen, and K. J. Maløy, Europhys. Lett. 92, 44001 (2010).

[8] J. Kim, S.-G. Je, and S.-B. Choe, Appl. Phys. Express 8, 063001 (2015).

[9] S. S. P. Parkin, M. Hayashi, and L. Thomas, Science 320, 190 (2008).

[10] P. Paruch and P. Ghosez, Nature (London) 534, 331 (2016).

[11] S. Lemerle, J. Ferré, C. Chappert, V. Mathet, T. Giamarchi, and P. Le Doussal, Phys. Rev. Lett. 80, 849 (1998).

[12] A. B. Kolton, A. Rosso, T. Giamarchi, and W. Krauth, Phys. Rev. B 79, 184207 (2009).

[13] E. E. Ferrero, L. Foini, T. Giamarchi, A. B. Kolton, and A. Rosso, Phys. Rev. Lett. 118, 147208 (2017).
[14] L. Thevenard, C. Gourdon, S. Haghgoo, J.-P. Adam, H. J. von Bardeleben, A. Lemaître, W. Schoch, and A. Thiaville, Phys. Rev. B 83, 245211 (2011).

[15] G. Durin and S. Zapperi, Phys. Rev. Lett. 84, 4705 (2000).

[16] G. Durin, F. Bohn, M. A. Corrêa, R. L. Sommer, P. Le Doussal, and K. J. Wiese, Phys. Rev. Lett. 117, 087201 (2016).

[17] A. Rosso and W. Krauth, Phys. Rev. Lett. 87, 187002 (2001).

[18] A. Lemaître, A. Miard, L. Travers, O. Mauguin, L. Largeau, C. Gourdon, V. Jeudy, M. Tran, and J.-M. George, Appl. Phys. Lett. 93, 021123 (2008).

[19] A. Dourlat, V. Jeudy, A. Lemaître, and C. Gourdon, Phys. Rev. B 78, 161303(R) (2008).

[20] P. J. Metaxas, J. P. Jamet, A. Mougin, M. Cormier, J. Ferré, V. Baltz, B. Rodmacq, B. Dieny, and R. L. Stamps, Phys. Rev. Lett. 99, 217208 (2007).

[21] J. Gorchon, S. Bustingorry, J. Ferré, V. Jeudy, A. B. Kolton, and T. Giamarchi, Phys. Rev. Lett. 113, 027205 (2014).

[22] V. Jeudy, R. Díaz Pardo, W. Savero Torres, S. Bustingorry, and A. B. Kolton, Phys. Rev. B 98, 054406 (2018).

[23] P. Paruch, A. B. Kolton, X. Hong, C. H. Ahn, and T. Giamarchi, Phys. Rev. B 85, 214115 (2012).

[24] A. Dourlat, V. Jeudy, C. Testelin, F. Bernardot, K. Khazen, C. Gourdon, L. Thevenard, L. Largeau, O. Mauguin, and A. Lemaître, J. Appl. Phys. 102, 023913 (2007).

[25] A. Rosso, A. K. Hartmann, and W. Krauth, Phys. Rev. E 67, 021602 (2003).

[26] S. F. Edwards and D. R. Wilkinson, Proc. R. Soc. London, Ser. A 381, 17 (1982). 